\title{
Multiple bifurcations and spatiotemporal patterns for a coupled two-cell Brusselator model
}

\author{
Wenjie Zuo and Junjie Wei \\ Communicated by Yuncheng You, received August 31, 2010.
}

\begin{abstract}
A coupled two-cell Brusselator model with diffusion effect subject to Neumann boundary condition is considered. Hopf bifurcations and global steady state bifurcations which bifurcate from the unique positive constant equilibrium point are investigated in detail. Meanwhile, Turing instability occurs when diffusion is present. Particularly, we show the existence of spatially inhomogeneous periodic solutions and non-constant steady state solutions, which exhibit rich spatiotemporal patterns in this coupled Brusselator system. Some numerical simulations are presented to illustrate the theoretical results obtained.
\end{abstract}

\section{Contents}

1. Introduction 363

2. Hopf bifurcation $\quad 365$

3. Steady state bifurcation $\quad 374$

References $\quad 383$

\section{Introduction}

It is well-known that the reaction-diffusion system with autocatalytic such as the Brusselator equations and the Gray-Scott equations $[\mathbf{1}, \mathbf{2}]$ can exhibit rich spatial patterns (including but not restricted to Turing patterns) $[\mathbf{3}, \mathbf{4}, \mathbf{5}, \mathbf{6 ,} \mathbf{7}]$. One of the most widely studied models is the Brusselator system, which describes the following chemical reactions:

$$
A \rightarrow U, B+U \rightarrow V+D, 2 U+V \rightarrow 3 U, U \rightarrow E,
$$

1991 Mathematics Subject Classification. 35; 37.

Key words and phrases. Coupled Brusselator model; diffusion; Hopf bifurcation; global steady state bifurcation; spatiotemporal patterns.

This research is supported by the National Natural Science Foundation of China (No. $11031002)$ 
where $A, B, D, E, U$ and $V$ are chemical reactants or products. Let $u(x, t)$ and $v(x, t)$ be the concentrations of $U$ and $V$, and assume that the concentrations of the input compounds $A$ and $B$ are held constants during the reaction process, denoted by $a$ and $b$, respectively. Thus one can obtain (diffusive) Brusselator equations:

$$
\left\{\begin{array}{l}
\frac{\partial u}{\partial t}=d_{1} \Delta u-(b+1) u+u^{2} v+a, \\
\frac{\partial v}{\partial t}=d_{2} \Delta v+b u-u^{2} v .
\end{array}\right.
$$

The model (1.1) has been studied by several researchers $(\operatorname{see}[\mathbf{8}, \mathbf{9}, \mathbf{1 0}, \mathbf{1 1}])$ in view of bifurcation.

The study of two-cell model of two coupled components is a substantial advance from one-cell model of two-component reaction-diffusion systems $[\mathbf{1 2}, \mathbf{1 3}]$. Coupled cells with diffusive reaction and mutual exchange are often adopted to describe the processes in living cells and tissues, or in distributed chemical reactions $[\mathbf{1 4}, \mathbf{1 5}]$.

In the present paper, we mainly consider a coupled Brusselator model, i.e. $(1.2)$

$$
\left\{\begin{array}{l}
\frac{\partial u_{1}}{\partial t}=d_{1} \triangle u_{1}+c\left(u_{3}-u_{1}\right)+a-(b+1) u_{1}+u_{1}^{2} u_{2},(x, t) \in \Omega \times(0,+\infty), \\
\frac{\partial u_{2}}{\partial t}=d_{2} \triangle u_{2}+b u_{1}-u_{1}^{2} u_{2},(x, t) \in \Omega \times(0,+\infty), \\
\frac{\partial u_{3}}{\partial t}=d_{1} \triangle u_{3}+c\left(u_{1}-u_{3}\right)+a-(b+1) u_{3}+u_{3}^{2} u_{4}, \quad(x, t) \in \Omega \times(0,+\infty), \\
\frac{\partial u_{4}}{\partial t}=d_{2} \triangle u_{4}+b u_{3}-u_{3}^{2} u_{4},(x, t) \in \Omega \times(0,+\infty), \\
\partial_{\nu} u_{i}=0, \text { on }(x, t) \in \partial \Omega \times(0,+\infty), i=1,2,3,4, \\
u_{i}(x, 0)=u_{i}(x) \geq 0, i=1,2,3,4,
\end{array}\right.
$$

where $\Omega \subset R^{N}, N \geq 1$ is a bounded domain with smooth boundary $\partial \Omega, d_{1}, d_{2}, a, b, c$ are positive constants, $\nu$ is outward unit normal vector on $\partial \Omega$, and no flux boundary condition is imposed, which implies that the system (1.2) is a closed one and has no flux across the boundary $\partial \Omega$. The system (1.2) has been studied by several researchers. For example, You [16] proved the existence of a global attractor of solution semi-flow of system (1.2) with Dirichlet boundary condition.

Recently, Zhou and $\mathrm{Mu}[\mathbf{1 7}]$ considered the existence and non-existence of positive non-trivial solutions of system (1.2) by utilizing the bifurcation theory and degree theory. Their theoretical analysis shows that the diffusion rate of this reaction and the size of the reactor play decisive roles in leading to the formation of stationary patterns.

Yi, Wei and Shi $[\mathbf{1 8}, \mathbf{1 9}]$ have investigated the Hopf and steady state bifurcations in a diffusion predator-prey model, and the multiple bifurcations in a diffusive bimolecular model, respectively.

Intriguing by the above work, for system (1.2), we shall choose the parameter $b$ as the main bifurcation parameter to study Hopf bifurcation and global steady state bifurcations, which bifurcate from the unique constant positive equilibrium $\left(a, \frac{b}{a}, a, \frac{b}{a}\right)$ of the system. Turing instability occurs when $d_{1}, d_{2}$ are in some certain region. These results suggest the existence of spatially inhomogeneous periodic orbits and the non-constant steady state solutions, which implies rich spatiotemporal patterns of a coupled Brusselator model. Finally, we discuss the interaction between Hopf bifurcation and steady state bifurcation. In the different parameter ranges, the intertwining of the two type of bifurcations is delicate. This provides some theoretical evidences for the complex dynamical behavior.

The rest of the paper is organized as follows. In Section 2, the stability and Hopf bifurcation analysis of the system (1.2) are considered. The Hopf bifurcation for 
general reaction-diffusion system(R-D system) consisting of $m$ equations is derived in Section 2.1, and these results are applied to Hopf bifurcation analysis of (1.2). In Section 3, global steady state bifurcation and interaction between Hopf bifurcation and steady state bifurcation are investigated. One longer proof is given in Appendix.

\section{Hopf bifurcation}

For convenience, we restrict ourselves to one-dimensional spatial domain $(0, l \pi)$, for which the structure of the eigenvalues is clear.

2.1. Hopf bifurcation for general R-D system. We consider a general R-D system subject to the homogeneous Neumann boundary condition:

$$
\begin{cases}\frac{\partial u_{i}(x, t)}{\partial t} & =d_{i} \Delta u_{i}(x, t)+f^{(i)}\left(b, u_{1}, \cdots, u_{m}\right), x \in(0, l \pi), t>0 \\ \frac{\partial u_{i}(x, t)}{\partial x} & =0, x=0, l \pi, t>0 \\ u_{i}(x, 0) & =u_{i}(x)>0, x \in(0, l \pi) \\ i & =1,2, \cdots, m\end{cases}
$$

where $d_{1}, d_{2}, \cdots, d_{m}$ are positive constants, and $f^{(i)}: R \times R^{m} \rightarrow R$ are $C^{k}(k \geq 3)$ with $f^{(i)}(b, 0, \cdots, 0) \equiv 0, i=1,2, \cdots, m$.

Denote

$$
U=\left(u_{1}, u_{2}, \cdots, u_{m}\right)^{T}, D=\left(\begin{array}{cccc}
d_{1} & & & \\
& d_{2} & & \\
& & \ddots & \\
& & & d_{m}
\end{array}\right)
$$

and $F(b, U)=\left(f^{(1)}, f^{(2)}, \cdots, f^{(m)}\right)$. Then $(2.1)$ can be rewritten as

$$
\frac{d U}{d t}=D \triangle U+F(b, U)
$$

Define the real-value Sobolev space

$$
X:=\left\{\left(u_{1}, \cdots, u_{m}\right) \in\left(H^{2}(0, l \pi)\right)^{m} \mid \frac{\partial u_{i}}{\partial x}(0, t)=\frac{\partial u_{i}}{\partial x}(l \pi, t)=0, i=1, \cdots, m\right\},
$$

and let the complexification of $X$ be $X_{c}:=X \oplus i X=\left\{x_{1}+i x_{2} \mid x_{1}, x_{2} \in X\right\}$. The linearized operator of the steady state system of $(2.1)$ evaluated at $(b, \mathbf{0})$ is

$$
L(b)=D \frac{\partial^{2}}{\partial x^{2}}+\left.J(F)\right|_{U \equiv \mathbf{0}}
$$

where $J(F)$ denotes the Jacobi matrix of $F$.

To consider Hopf bifurcation, we assume that the following condition holds for some $b_{0} \in R$.

(H1) There exists a neighborhood $O$ of $b_{0}$ such that for $b \in O, L(b)$ has a pair of conjugate eigenvalues $\alpha(b) \pm i \omega(b)$, continuously differentiable in $b$, with $\alpha\left(b_{0}\right)=0, \omega\left(b_{0}\right)=\omega_{0}$ and $\alpha^{\prime}\left(b_{0}\right) \neq 0$, and all other eigenvalues of $L(b)$ have non-zero real parts for $b \in O$.

It is well-known that the eigenvalue problem

$$
\left\{\begin{array}{l}
-\varphi^{\prime \prime}=\mu \varphi, x=(0, l \pi), \\
\varphi^{\prime}(0)=\varphi^{\prime}(l \pi)=0,
\end{array}\right.
$$


has eigenvalues $\mu_{n}=\frac{n^{2}}{l^{2}}(n=0,1,2, \cdots)$, with corresponding eigenfunctions $\cos \frac{n}{l} x$. Let

$$
\Phi:=\left(\phi_{1}, \phi_{2}, \cdots, \phi_{m}\right)^{T}=\sum_{n=0}^{\infty} \cos \frac{n}{l} x\left(a_{1 n}, a_{2 n}, \cdots, a_{m n}\right)^{T}
$$

be an eigenfunction for $L(b)$ with eigenvalue $\beta(b)$, that is, $L(b) \Phi=\beta(b) \Phi$. Then from a straightforward computation, we obtain

$$
L_{n}(b)\left(a_{1 n}, a_{2 n}, \cdots, a_{m n}\right)^{T}=\beta(b)\left(a_{1 n}, a_{2 n}, \cdots, a_{m n}\right)^{T},
$$

where $L_{n}(b)=D\left(-\frac{n^{2}}{l^{2}}\right)+\left.J(F)\right|_{U \equiv \mathbf{0}}$. We adopt the framework of $[\mathbf{1 8}, \mathbf{2 0}]$, and rewrite $(2.2)$ in the abstract form:

$$
\frac{d U}{d t}=L(b) U+G(b, U),
$$

where $G(b, U)=F(b, U)-\left.J(F)\right|_{U \equiv \mathbf{0}}$. At $b=b_{0}$, the system (2.7) is reduced to

$$
\frac{d U}{d t}=L\left(b_{0}\right) U+G\left(b_{0}, U\right) \text {. }
$$

Let $\langle\cdot, \cdot\rangle$ be the complex-valued $L^{2}$ inner product on Hilbert space $X_{c}$, defined as

$$
\left\langle U_{1}, U_{2}\right\rangle=\int_{0}^{l \pi}\left(\bar{u}_{1} v_{1}+\bar{u}_{2} v_{2}+\cdots+\bar{u}_{m} v_{m}\right) d x
$$

with $U_{1}=\left(u_{1}, u_{2}, \cdots, u_{m}\right)^{T}, U_{2}=\left(v_{1}, v_{2}, \cdots, v_{m}\right)^{T} \in X_{c}$. Denote by $L^{*}\left(b_{0}\right)$ the adjoint operator of $L\left(b_{0}\right)$, from (H1), we can choose

$$
q:=\cos \frac{n x}{l}\left(a_{1 n}, a_{2 n}, \cdots, a_{m n}\right)^{T}, q^{*}:=\cos \frac{n x}{l}\left(a_{1 n}^{*}, a_{2 n}^{*}, \cdots, a_{m n}^{*}\right)^{T} \in X_{c},
$$

so that

$$
L\left(b_{0}\right) q=i \omega_{0} q, L^{*}\left(b_{0}\right) q^{*}=-i \omega_{0} q^{*},\left\langle q^{*}, q\right\rangle=1, \text { and }\left\langle q^{*}, \bar{q}\right\rangle=0 .
$$

We decompose $X=X^{c} \oplus X^{s}$ with $X^{c}:=\{z q+\bar{z} \bar{q} \mid z \in C\}, X^{s}:=\left\{v \in X \mid\left\langle q^{*}, v\right\rangle=\right.$ $0\}$. For any $U \in X$, there exist $z \in C$ and $W=\left(w_{1}, w_{2}, \cdots, w_{m}\right) \in X^{s}$ such that

$$
U=z q+\bar{z} \bar{q}+W
$$

$$
u_{i}=z a_{i n} \cos \frac{n x}{l}+\bar{z} \bar{a}_{i n} \cos \frac{n x}{l}+w_{i}, i=1,2, \cdots, m .
$$

Thus (2.7) is reduced to the following system:

$$
\left\{\begin{array}{l}
\frac{d z}{d t}=i \omega_{0} z+\left\langle q^{*}, G_{0}\right\rangle \\
\frac{d W}{d t}=L\left(b_{0}\right) W+H(z, \bar{z}, W)
\end{array}\right.
$$

where $G_{0}=G\left(b_{0}, U\right)$,

$$
H(z, \bar{z}, W):=G_{0}-\left\langle q^{*}, G_{0}\right\rangle q-\overline{\left\langle q^{*}, G_{0}\right\rangle} \bar{q} .
$$

As in $[\mathbf{1 8}, \mathbf{2 0}]$, we write $G_{0}$ in the following form:

$$
G_{0}(U)=\frac{1}{2} Q(U, U)+\frac{1}{6} C(U, U, U)+O\left(|U|^{4}\right),
$$

where $Q$ and $C$ are symmetric multilinear forms. Denote $Q_{X Y}=Q(X, Y)$ and $C_{X Y Z}=C(X, Y, Z)$. For later uses, we calculate $Q_{q q}, Q_{q \bar{q}}$ and $C_{q q \bar{q}}$ as follows.

$$
Q_{q q}=\cos ^{2} \frac{n x}{l}\left(b_{1 n}, b_{2 n}, \cdots, b_{m n}\right)^{T}, Q_{q \bar{q}}=\cos ^{2} \frac{n x}{l}\left(c_{1 n}, c_{2 n}, \cdots, c_{m n}\right)^{T},
$$




$$
C_{q q \bar{q}}=\cos ^{3} \frac{n x}{l}\left(d_{1 n}, d_{2 n}, \cdots, d_{m n}\right)^{T},
$$

where

$$
\begin{aligned}
b_{i n}= & \sum_{k=1}^{m} f_{u_{k} u_{k}}^{(i)}\left(b_{0}, \mathbf{0}\right) a_{k n}^{2}+2 \sum_{k=1}^{m-1} \sum_{j=k+1}^{m} f_{u_{k} u_{j}}^{(i)}\left(b_{0}, \mathbf{0}\right) a_{k n} a_{j n}, \\
c_{i n}= & \sum_{k=1}^{m} f_{u_{k} u_{k}}^{(i)}\left(b_{0}, \mathbf{0}\right)\left|a_{k n}\right|^{2}+\sum_{k=1}^{m-1} \sum_{j=k+1}^{m} f_{u_{k} u_{j}}^{(i)}\left(b_{0}, \mathbf{0}\right)\left(a_{k n} \bar{a}_{j n}+\bar{a}_{k n} a_{j n}\right), \\
d_{i n}= & \sum_{k=1}^{m} f_{u_{k} u_{k} u_{k}}^{(i)}\left(b_{0}, \mathbf{0}\right)\left|a_{k n}\right|^{2} a_{k n}+\sum_{k=1}^{m-1} \sum_{j=k+1}^{m} f_{u_{k} u_{k} u_{j}}^{(i)}\left(b_{0}, \mathbf{0}\right)\left(a_{k n}^{2} \bar{a}_{j n}+2\left|a_{k n}\right|^{2} a_{j n}\right) \\
& +2 \sum_{k=1}^{m-2} \sum_{j=k+1}^{m-1} \sum_{l=j+1}^{m} f_{u_{k} u_{j} u_{l}}^{(i)}\left(b_{0}, \mathbf{0}\right)\left(a_{k n} a_{j n} \bar{a}_{l n}+a_{k n} \bar{a}_{j n} a_{l n}+\bar{a}_{k n} a_{j n} a_{l n}\right), \\
& i=1,2, \cdots, m .
\end{aligned}
$$

Let

$$
H(z, \bar{z}, W)=H_{20} \frac{z^{2}}{2}+H_{11} z \bar{z}+H_{02} \frac{\bar{z}^{2}}{2}+\cdots .
$$

Then by (2.13) and (2.14), we have

$$
\left\{\begin{array}{l}
H_{20}=Q_{q q}-\left\langle q^{*}, Q_{q q}\right\rangle q-\left\langle\bar{q}^{*}, Q_{q q}\right\rangle \bar{q} \\
H_{11}=Q_{q \bar{q}}-\left\langle q^{*}, Q_{q \bar{q}}\right\rangle q-\left\langle\bar{q}^{*}, Q_{q \bar{q}}\right\rangle \bar{q}
\end{array}\right.
$$

Let

$$
W=W_{20}(\theta) \frac{z^{2}}{2}+W_{11}(\theta) z \bar{z}+W_{02}(\theta) \frac{\bar{z}^{2}}{2}+\cdots .
$$

By (2.15) and together with $L\left(b_{0}\right) W+H(z, \bar{z}, W)=\frac{d W}{d t}=\frac{\partial W}{\partial z} \frac{d z}{d t}+\frac{\partial W}{\partial \bar{z}} \frac{d \bar{z}}{d t}$, we have

$$
W_{20}=\left[2 i \omega_{0} I-L\left(b_{0}\right)\right]^{-1} H_{20} \text { and } W_{11}=-\left[L^{-1}\left(b_{0}\right)\right] H_{11} \text {. }
$$

We notice $\int_{0}^{l \pi} \cos ^{3} \frac{n x}{l} d x=0, \forall n \in N=\{1,2, \cdots\}$, and by calculation,

$$
\left\langle q^{*}, Q_{q q}\right\rangle=\left\langle q^{*}, Q_{q \bar{q}}\right\rangle=\left\langle\bar{q}^{*}, Q_{q q}\right\rangle=\left\langle\bar{q}^{*}, Q_{\bar{q} \bar{q}}\right\rangle=0 .
$$

Then by (2.16), (2.18) and (2.19), we have

$$
\begin{aligned}
& W_{20}=\left\{\begin{array}{lr}
\frac{1}{2}\left[2 i \omega_{0} I-L\left(b_{0}\right)\right]^{-1}\left[\left(\cos \frac{2 n x}{l}+1\right)\left(b_{1 n}, b_{2 n}, \cdots, b_{m n}\right)^{T}\right], & \text { if } n \in N, \\
{\left[2 i \omega_{0} I-L\left(b_{0}\right)\right]^{-1}\left[\left(b_{10}, b_{20}, \cdots, b_{m 0}\right)^{T}\right.} & \text { if } n=0, \\
\left.-2 \operatorname{Re}\left(\left\langle q^{*}, Q_{q q}\right\rangle\left(a_{10}, a_{20}, \cdots, a_{m 0}\right)^{T}\right)\right], & \text { if } n \in N,
\end{array}\right. \\
& W_{11}= \begin{cases}-\frac{1}{2} L^{-1}\left(b_{0}\right)\left[\left(\cos \frac{2 n x}{l}+1\right)\left(c_{1 n}, c_{2 n}, \cdots, c_{m n}\right)^{T}\right], \\
-L^{-1}\left(b_{0}\right)\left[\left(c_{10}, c_{20}, \cdots, c_{m 0}\right)^{T}\right. \\
\left.-2 \operatorname{Re}\left(\left\langle q^{*}, Q_{q \bar{q}}\right\rangle\left(a_{10}, a_{20}, \cdots, a_{m 0}\right)^{T}\right)\right], & \text { if } n=0 .\end{cases}
\end{aligned}
$$

Therefore the reaction-diffusion system restricted to the center manifold is given by

$$
\frac{d z}{d t}=i \omega_{0} z+\left\langle q^{*}, G_{0}\right\rangle=i \omega_{0} z+\sum_{2 \leq i+j \leq 3} \frac{g_{i j}}{i ! j !} z^{i} \bar{z}^{j}+\cdots
$$


where $g_{20}=\left\langle q^{*}, Q_{q q}\right\rangle, g_{11}=\left\langle q^{*}, Q_{q \bar{q}}\right\rangle, g_{02}=\left\langle q^{*}, Q_{\bar{q} \bar{q}}\right\rangle$, and

$$
\begin{aligned}
& g_{21}=2\left\langle q^{*}, Q_{W_{11} q}\right\rangle+\left\langle q^{*}, Q_{W_{20} \bar{q}}\right\rangle+\left\langle q^{*}, C_{q q \bar{q}}\right\rangle, \\
& c_{1}\left(b_{0}\right)=\frac{i}{2 \omega_{0}}\left(g_{20} g_{11}-2\left|g_{11}\right|^{2}-\frac{1}{3}\left|g_{02}\right|^{2}\right)+\frac{g_{21}}{2},
\end{aligned}
$$

which determine the properties of bifurcating periodic solutions at the critical value $b_{0}$. We have the following Hopf bifurcation theorem for the general R-D system (2.1).

THEOREM 2.1. Suppose (H1)is satisfied. Then (2.1) possesses a family of realvalued $T(s)$-periodic solutions $\left(b(s), u_{1}(s)(x, t), \cdots, u_{m}(s)(x, t)\right)$, for $s$ sufficiently small,

$$
\left(u_{1}(s)(x, t), \cdots, u_{m}(s)(x, t)\right)
$$

can be parameterized in the following form:

$$
\left\{\begin{array}{l}
u_{1}=s\left(a_{1 n} e^{2 \pi i t / T(s)} \cos \frac{n x}{l}+\bar{a}_{1 n} e^{-2 \pi i t / T(s)} \cos \frac{n x}{l}\right)+o(s), \\
\cdots \\
u_{m}=s\left(a_{m n} e^{2 \pi i t / T(s)} \cos \frac{n x}{l}+\bar{a}_{m n} e^{-2 \pi i t / T(s)} \cos \frac{n x}{l}\right)+o(s) .
\end{array}\right.
$$

Furthermore:

1. The Hopf bifurcation is forward (backward) if

$$
\mu_{2}=-\frac{\operatorname{Re}\left(c_{1}\left(b_{0}\right)\right)}{\alpha^{\prime}\left(b_{0}\right)}>0(<0) .
$$

2. The bifurcating periodic solutions on the center manifold are orbitally asymptotically stable (unstable) if Re $\left(c_{1}\left(b_{0}\right)\right)<0(>0)$. Particularly, if all other eigenvalues of $L\left(b_{0}\right)$ have negative real parts, then the bifurcating periodic solutions are orbitally asymptotically stable (unstable) if $\operatorname{Re}\left(c_{1}\left(b_{0}\right)\right)<0(>0)$.

2.2. Stability and Hopf bifurcation analysis in a coupled Brusselator model. Straightforward calculation shows that the system (1.2) has a unique constant fixed point $U^{*}=\left(a, \frac{b}{a}, a, \frac{b}{a}\right)$. To cast our discussion into the framework of Section 2.1, we translate (1.2) into the following system by the translation

$$
\bar{u}_{1}=u_{1}-a, \bar{u}_{2}=u_{2}-\frac{b}{a}, \bar{u}_{3}=u_{3}-a, \bar{u}_{4}=u_{4}-\frac{b}{a},
$$

and still let $u_{i}(i=1,2,3,4)$ replace $\bar{u}_{i}(i=1,2,3,4)$, respectively.

$$
\left\{\begin{array}{l}
\frac{\partial u_{1}}{\partial t}=d_{1} \triangle u_{1}+(b-c-1) u_{1}+a^{2} u_{2}+c u_{3}+u_{1}^{2} u_{2}+\frac{b}{a} u_{1}^{2}+2 a u_{1} u_{2} \\
\frac{\partial u_{2}}{\partial t}=d_{2} \triangle u_{2}-b u_{1}-a^{2} u_{2}-u_{1}^{2} u_{2}-\frac{b}{a} u_{1}^{2}-2 a u_{1} u_{2}, \\
\frac{\partial u_{3}}{\partial t}=d_{1} \triangle u_{3}+c u_{1}+(b-c-1) u_{3}+a^{2} u_{4}+u_{3}^{2} u_{4}+\frac{b}{a} u_{3}^{2}+2 a u_{3} u_{4} \\
\frac{\partial u_{4}}{\partial t}=d_{2} \triangle u_{4}-b u_{3}-a^{2} u_{4}-u_{3}^{2} u_{4}-\frac{b}{a} u_{3}^{2}-2 a u_{3} u_{4} \\
\frac{\partial u_{i}}{\partial x}(0, t)=\frac{\partial u_{i}}{\partial x}(l \pi, t)=0, t>0, i=1,2,3,4
\end{array}\right.
$$

Denote the linearized operator of the steady state of $(2.23)$ evaluated in $(0,0,0,0)$ by

$$
L(b):=\left(\begin{array}{cccc}
d_{1} \frac{\partial^{2}}{\partial x^{2}}+b-c-1 & a^{2} & c & 0 \\
-b & d_{2} \frac{\partial^{2}}{\partial x^{2}}-a^{2} & 0 & 0 \\
c & 0 & d_{1} \frac{\partial^{2}}{\partial x^{2}}+b-c-1 & a^{2} \\
0 & 0 & -b & d_{2} \frac{\partial^{2}}{\partial x^{2}}-a^{2}
\end{array}\right),
$$


and

$$
L_{n}(b)=\left(\begin{array}{cccc}
-\frac{d_{1} n^{2}}{l^{2}}+b-c-1 & a^{2} & c & 0 \\
-b & -\frac{d_{2} n^{2}}{l^{2}}-a^{2} & 0 & 0 \\
c & 0 & -\frac{d_{1} n^{2}}{l^{2}}+b-c-1 & a^{2} \\
0 & 0 & -b & -\frac{d_{2} n^{2}}{l^{2}}-a^{2}
\end{array}\right) .
$$

The characteristic equation of $L_{n}(b)$ is given by

$$
\left[\lambda^{2}-T_{n}(b) \lambda+D_{n}(b)\right]\left[\lambda^{2}-P_{n}(b) \lambda+Q_{n}(b)\right]=0,
$$

where

$$
\left\{\begin{array}{l}
T_{n}(b)=-\frac{\left(d_{1}+d_{2}\right) n^{2}}{l^{2}}-a^{2}+b-1 \\
D_{n}(b)=\left(\frac{d_{2} n^{2}}{l^{2}}+a^{2}\right)\left(\frac{d_{1} n^{2}}{l^{2}}-b+1\right)+b a^{2} \\
P_{n}(b)=-\frac{\left(d_{1}+d_{2}\right) n^{2}}{l^{2}}-a^{2}+b-2 c-1 \\
Q_{n}(b)=\left(\frac{d_{2} n^{2}}{l^{2}}+a^{2}\right)\left(\frac{d_{1} n^{2}}{l^{2}}-b+2 c+1\right)+b a^{2}
\end{array}\right.
$$

Obviously, $\lambda_{i}$ is an eigenvalue of $L_{n}(b)$ on $X$ if and only if $\lambda_{i}$ is a root of the following equations (2.26) or (2.27).

$$
\begin{aligned}
& \lambda^{2}-T_{n}(b) \lambda+D_{n}(b)=0, \\
& \lambda^{2}-P_{n}(b) \lambda+Q_{n}(b)=0 .
\end{aligned}
$$

Now we shall identify all the possible Hopf bifurcation values $b_{0}$ which satisfy the condition (H1). We carry it out in three steps.

Step 1. We shall find these $b_{0}$, such that at $b=b_{0}$, Eq.(2.26) has a pair of simple, pure imaginary roots, and all other roots of Eq.(2.26) have non-zero real parts. That is, there exists $n \in N \cup\{0\}$, such that

$$
T_{n}\left(b_{0}\right)=0, D_{n}\left(b_{0}\right)>0, T_{j}\left(b_{0}\right) \neq 0, D_{j}\left(b_{0}\right) \neq 0, \text { for } \forall j \neq n .
$$

If $T_{n}(b)=0$, we have $b_{n,+}^{H}=\frac{\left(d_{1}+d_{2}\right) n^{2}}{l^{2}}+a^{2}+1$, and

$$
D_{n}\left(b_{n,+}^{H}\right)=-d_{2}^{2}\left(\frac{n^{2}}{l^{2}}\right)^{2}+a^{2}\left(d_{1}-d_{2}\right) \frac{n^{2}}{l^{2}}+a^{2} .
$$

In order that $D_{n}\left(b_{n,+}^{H}\right)>0$, we need to prove the following inequality

$$
d_{2}^{2}\left(\frac{n^{2}}{l^{2}}\right)^{2}-a^{2}\left(d_{1}-d_{2}\right) \frac{n^{2}}{l^{2}}-a^{2}<0
$$

or equivalently,

$$
\frac{a^{2}\left(d_{1}-d_{2}\right)-\sqrt{A}}{2 d_{2}^{2}}<\frac{n^{2}}{l^{2}}<\frac{a^{2}\left(d_{1}-d_{2}\right)+\sqrt{A}}{2 d_{2}^{2}},
$$

where $A=a^{4}\left(d_{1}-d_{2}\right)^{2}+4 a^{2} d_{2}^{2}$, that is,

$$
0 \leq n^{2}<\frac{a^{2}\left(d_{1}-d_{2}\right)+\sqrt{a^{4}\left(d_{1}-d_{2}\right)^{2}+4 a^{2} d_{2}^{2}}}{2 d_{2}^{2}} l^{2} .
$$

Obviously,

$$
T_{j}\left(b_{n,+}^{H}\right)=-\frac{d_{1}+d_{2}}{l^{2}}\left(j^{2}-n^{2}\right) \neq 0, \forall j \neq n .
$$


To let $D_{j}\left(b_{n,+}^{H}\right) \neq 0, \forall j \neq n$, we need

$$
\frac{\left(d_{1}+d_{2}\right) n^{2}}{l^{2}}+a^{2} \neq \frac{d_{1} j^{2}}{l^{2}}+\frac{a^{2} l^{2}}{d_{2} j^{2}}+\frac{d_{1} a^{2}}{d_{2}}, n \neq j, \forall j \in N .
$$

In this case, all the possible parameters $b$ can be labeled as

$$
\Lambda_{1}^{H}=\left\{b_{n,+}^{H}, n=0,1, \cdots, N, \text { and } n \text { satisfies }(2.29)\right\},
$$

for some $N \in N \cup\{0\}$, satisfying

$$
a^{2}+1=b_{0,+}^{H}<b_{1,+}^{H}<\cdots<b_{N,+}^{H}<+\infty,
$$

such that

$$
0 \leq \frac{b_{N,+}^{H}-1-a^{2}}{d_{1}+d_{2}}<\frac{a^{2}\left(d_{1}-d_{2}\right)+\sqrt{a^{4}\left(d_{1}-d_{2}\right)^{2}+4 a^{2} d_{2}^{2}}}{2 d_{2}^{2}} .
$$

Step 2. We shall find these $b_{0}$ at which Eq.(2.27) has a pair of simple, pure imaginary roots, and all other roots of Eq.(2.27) have non-zero real parts.

If $P_{n}(b)=0$, we have $b_{n,-}^{H}=\frac{\left(d_{1}+d_{2}\right) n^{2}}{l^{2}}+a^{2}+2 c+1$.

To $Q_{j}\left(b_{n,-}^{H}\right) \neq 0$, we have

$$
\frac{\left(d_{1}+d_{2}\right) n^{2}}{l^{2}}+a^{2} \neq \frac{d_{1} j^{2}}{l^{2}}+\frac{a^{2} l^{2}(2 c+1)}{d_{2} j^{2}}+\frac{d_{1} a^{2}}{d_{2}}, n \neq j, \forall j \in N .
$$

Similar to Step 1, we can derive the following results.

In this case, all the possible parameters $b$ can be labeled as

$$
\Lambda_{2}^{H}=\left\{b_{n,-}^{H}, \text { and } n=0,1, \cdots, N^{\prime} \text {, and } n \text { satisfies (2.31) }\right\},
$$

for some $N^{\prime} \in N \cup\{0\}$, satisfying

$$
a^{2}+2 c+1=b_{0,-}^{H}<b_{1,-}^{H}<\cdots<b_{N^{\prime},-}^{H}<+\infty,
$$

such that

$$
0 \leq \frac{b_{N^{\prime},-}^{H}-a^{2}-2 c-1}{d_{1}+d_{2}}<\frac{a^{2}\left(d_{1}-d_{2}\right)+\sqrt{a^{4}\left(d_{1}-d_{2}\right)^{2}+4 a^{2} d_{2}^{2}(2 c+1)}}{2 d_{2}^{2}} .
$$

Step 3. The transversality condition holds.

Eq.(2.26) has a pair of conjugate roots $\alpha_{1}(b) \pm i \omega_{1}(b)$, near $b=b_{n,+}^{H}$, where

$$
\alpha_{1}(b)=-\frac{\left(d_{1}+d_{2}\right) n^{2}}{2 l^{2}}-\frac{1}{2}\left(a^{2}+1\right)+\frac{b}{2}, \omega_{1}(b)=\sqrt{D_{n}(b)-\alpha_{1}^{2}(b)} .
$$

Clearly, $\left.\alpha_{1}^{\prime}(b)\right|_{b=b_{n,+}^{H}}=\frac{1}{2}>0$.

Eq.(2.27) has a pair of conjugate roots $\alpha_{2}(b) \pm i \omega_{2}(b)$, near $b=b_{n,-}^{H}$, where

$$
\alpha_{2}(b)=-\frac{\left(d_{1}+d_{2}\right) n^{2}}{2 l^{2}}-\frac{1}{2}\left(a^{2}+2 c+1\right)+\frac{b}{2}, \omega_{2}(b)=\sqrt{Q_{n}(b)-\alpha_{2}^{2}(b)} .
$$

Clearly, $\left.\alpha_{2}^{\prime}(b)\right|_{b=b_{n,-}^{H}}=\frac{1}{2}>0$. For convenience, we denote

$$
\Lambda^{H}=\Lambda_{1}^{H} \cup \Lambda_{2}^{H}=\left\{\left.b_{n,+}^{H}\right|_{n=0} ^{N}\right\} \cup\left\{\left.b_{n,-}^{H}\right|_{n=0} ^{N^{\prime}}\right\} \triangleq\left\{b_{n}^{H}\right\} .
$$

In addition, from $D_{j}(b)=0$, we have $b_{j,+}=\frac{d_{1} j^{2}}{l^{2}}+\frac{a^{2} l^{2}}{d_{2} j^{2}}+\frac{d_{1} a^{2}}{d_{2}}+1$. Then,

$$
D_{j}(b)>0 \text {, when } b<b_{j,+} \text { and } D_{j}(b)<0 \text {, when } b>b_{j,+} \text {. }
$$

Furthermore,

$$
D_{j}(b)>0, \forall j \in N=\{1,2, \cdots\} \text { when } b<\min _{j \in N}\left\{b_{j,+}\right\} .
$$


Meanwhile, $\min _{j \in N}\left\{b_{j,+}\right\}=\left\{\begin{array}{l}\left(1+\sqrt{\frac{d_{1}}{d_{2}}} a\right)^{2}, \text { if } \exists k \in N \text {, s.t. } k^{2}=\frac{a l^{2}}{\sqrt{d_{1} d_{2}}} \\ \min \left\{b_{k}, b_{k+1}\right\}, \text { if } \exists k \in N \text {, s.t. } k^{2}<\frac{a l^{2}}{\sqrt{d_{1} d_{2}}}<(k+1)^{2} .\end{array}\right.$

From the above analysis, we draw the following conclusions.

THEOREM 2.2. For the system (1.2), the following results are true:

(i) If $\min _{j \in N}\left\{b_{j,+}\right\}>1+a^{2}$, then the equilibrium point $\left(a, \frac{b}{a}, a, \frac{b}{a}\right)$ is asymptotically stable when $b \in\left(0, a^{2}+1\right)$, and unstable when $b \in\left(a^{2}+1,+\infty\right)$.

(ii) If $\min _{j \in N}\left\{b_{j,+}\right\}<1+a^{2}$, then the equilibrium point $\left(a, \frac{b}{a}, a, \frac{b}{a}\right)$ is asymptotically stable when $b \in\left(0, \min _{j \in N}\left\{b_{j,+}\right\}\right)$, and unstable when $b \in\left(\min _{j \in N}\left\{b_{j,+}\right\},+\infty\right)$. Thus Turing instability occurs.

(iii) The system (1.2) undergoes a Hopf bifurcation at $b=b_{n}^{H} \in \Lambda^{H}$ (defined above), and the bifurcating periodic solutions can be parameterized in the form of (2.21).

Moreover,

1. The bifurcating periodic solutions from $b=b_{0,+}^{H}=a^{2}+1$ are spatially homogenous, which coincides with the periodic solutions of the corresponding ODE system.

2. The bifurcating periodic solutions from $b=b_{n}^{H} \in \Lambda^{H} \backslash\left\{b_{0,+}^{H}\right\}$ are spatially inhomogeneous.

In what follows, we concentrate on the direction of bifurcation and stability of the bifurcating periodic solutions.

THEOREM 2.3. For the system (1.2), the Hopf bifurcation at $b=b_{0,+}^{H}=$ $a^{2}+1$ is forward, and the bifurcating periodic solutions are asymptotically stable if $\min _{j \in N}\left\{b_{j,+}\right\}>1+a^{2}$, and unstable if $\min _{j \in N}\left\{b_{j,+}\right\}<1+a^{2}$.

Proof. By Theorem 2.1, in order to determine the stability and direction of the bifurcating periodic solutions, we need to calculate $\operatorname{Rec}_{1}\left(b_{0,+}^{H}\right)$.

$$
\omega_{0}=\sqrt{D_{0}\left(b_{0,+}^{H}\right)}=\sqrt{a^{2}}=a>0, \text { if } b=b_{0,+}^{H}=a^{2}+1 .
$$

Let $q:=\left(a_{10}, a_{20}, a_{30}, a_{40}\right)^{T}=\left(-\frac{1}{b_{0,+}^{H}}\left(a^{2}+i a\right), 1,-\frac{1}{b_{0,+}^{H}}\left(a^{2}+i a\right), 1\right)^{T}$, and

$$
q^{*^{\prime}}:=\left(a_{10}^{*^{\prime}}, a_{20}^{*^{\prime}}, a_{30}^{*^{\prime}}, a_{40}^{*^{\prime}}\right)^{T}=\left(\frac{1}{a}(a-i), 1, \frac{1}{a}(a-i), 1\right)^{T}
$$

Let $q^{*}=D\left(\frac{1}{a}(a-i), 1, \frac{1}{a}(a-i), 1\right)^{T}$, where $\bar{D}=\left[\frac{4 l \pi}{a^{2}+1}(1-a i)\right]^{-1}$.

Recall

$$
\begin{aligned}
f^{(1)}\left(b, u_{1}, u_{2}, u_{3}, u_{4}\right) & =u_{1}^{2} u_{2}+\frac{b}{a} u_{1}^{2}+2 a u_{1} u_{2}=-f^{(2)}\left(b, u_{1}, u_{2}, u_{3}, u_{4}\right), \\
f^{(3)}\left(b, u_{1}, u_{2}, u_{3}, u_{4}\right) & =u_{3}^{2} u_{4}+\frac{b}{a} u_{3}^{2}+2 a u_{3} u_{4}=-f^{(4)}\left(b, u_{1}, u_{2}, u_{3}, u_{4}\right) .
\end{aligned}
$$


By Section 2.1, we can obtain

$$
\begin{aligned}
& b_{10}=-b_{20}=b_{30}=-b_{40}=\frac{2 b_{0,+}^{H}}{a} a_{10}^{2}+4 a a_{10} a_{20}=-2 a \\
& c_{10}=-c_{20}=c_{30}=-c_{40}=\frac{2 b_{0,+}^{H}}{a}\left|a_{10}\right|^{2}+2 a\left(a_{10} \bar{a}_{20}+\bar{a}_{10} a_{20}\right)=\frac{2 a\left(1-a^{2}\right)}{1+a^{2}} \\
& d_{10}=-d_{20}=d_{30}=-d_{40}=2\left(a_{10}^{2} \bar{a}_{20}+2\left|a_{10}\right|^{2} a_{20}\right)=\frac{6 a^{4}+2 a^{2}+4 a^{3} i}{\left(1+a^{2}\right)^{2}}
\end{aligned}
$$

and

$$
\begin{aligned}
& g_{20}=\left\langle q^{*}, Q_{q q}\right\rangle=-4 \bar{D} l \pi i=a-i, \\
& g_{11}=\left\langle q^{*}, Q_{q \bar{q}}\right\rangle=\frac{1-a^{2}}{1+a^{2}}(-a+i), \\
& \left\langle q^{*}, C_{q q \bar{q}}\right\rangle=\frac{-3 a^{2}+a i}{1+a^{2}} .
\end{aligned}
$$

By simple calculation, we can obtain

$$
\left\{\begin{array}{l}
H_{20}=\left(b_{10},-b_{10}, b_{10},-b_{10}\right)^{T}-2 \operatorname{Re}\left(\left\langle q^{*}, Q_{q q}\right\rangle\left(a_{10}, a_{20}, a_{30}, a_{40}\right)^{T}\right) \\
=(0,0,0,0)^{T} \\
H_{11}=\left(c_{10},-c_{10}, c_{10},-c_{10}\right)^{T}-2 \operatorname{Re}\left(\left\langle q^{*}, Q_{q \bar{q}}\right\rangle\left(a_{10}, a_{20}, a_{30}, a_{40}\right)^{T}\right) \\
=(0,0,0,0)^{T}
\end{array}\right.
$$

which implies that $W_{20}=W_{11}=0$. So

$$
\left\langle q^{*}, Q_{W_{11} q}\right\rangle=\left\langle q^{*}, Q_{W_{20} \bar{q}}\right\rangle=0
$$

Therefore

$$
\begin{aligned}
\operatorname{Rec}_{1}\left(b_{0,+}^{H}\right) & =\operatorname{Re}\left\{\frac{i}{2 \omega_{0}} g_{20} g_{11}+\frac{1}{2}\left\langle q^{*}, C_{q q \bar{q}}\right\rangle\right\} \\
& =\operatorname{Re}\left\{\frac{i}{2 a}(a-i) \frac{1-a^{2}}{1+a^{2}}(-a+i)+\frac{-3 a^{2}+a i}{2\left(1+a^{2}\right)}\right\} \\
& =\frac{i}{2 a} \frac{1-a^{2}}{1+a^{2}}(2 a i)+\frac{-3 a^{2}}{2\left(1+a^{2}\right)} \\
& =\frac{-a^{2}-2}{2\left(1+a^{2}\right)}<0 .
\end{aligned}
$$

Since $\alpha_{1}^{\prime}\left(b_{0,+}^{H}\right)=\frac{1}{2}>0$, and then by Theorem 2.1, the Hopf bifurcation is forward.

On the other hand, from (2.28) and (2.34), if $\min _{j \in N}\left\{b_{j,+}\right\}>1+a^{2}, T_{j}\left(b_{0,+}^{H}\right)<$ $0, D_{j}\left(b_{0,+}^{H}\right)>0$, for any $j \in\{1,2, \cdots\}$, and $P_{j}\left(b_{0,+}^{H}\right)<0, Q_{j}\left(b_{0,+}^{H}\right)>0$ for any $j \in\{0,1,2, \cdots\}$, so the bifurcating periodic solutions are asymptotically stable. If $\min _{j \in N}\left\{b_{j,+}\right\}<1+a^{2}$, set $\min _{j \in N}\left\{b_{j,+}\right\} \triangleq b_{k,+}$, there exists $k \in N$ such that $D_{k}\left(b_{0,+}^{H}\right)<0$, that is, (2.24) has at least a positive root. So the bifurcating periodic solutions are unstable. 

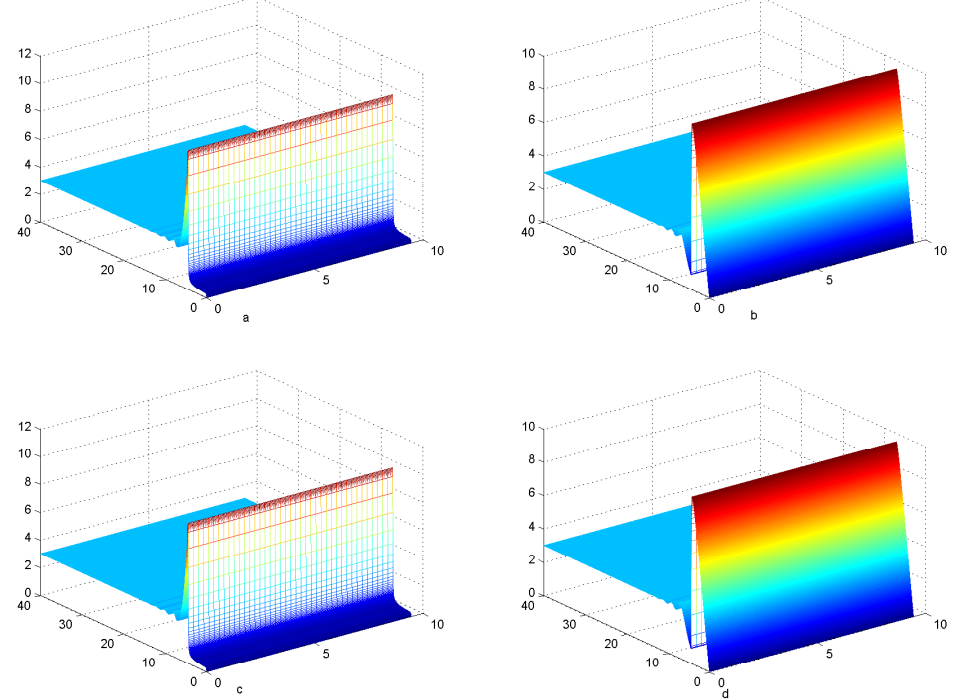

FIGURE 1 . The positive equilibrium is asymptotically stable when $b<a^{2}+1 \doteq 10$, where $a=3, b=9$, and initial data is $(4,2.8,4,2.8)$.
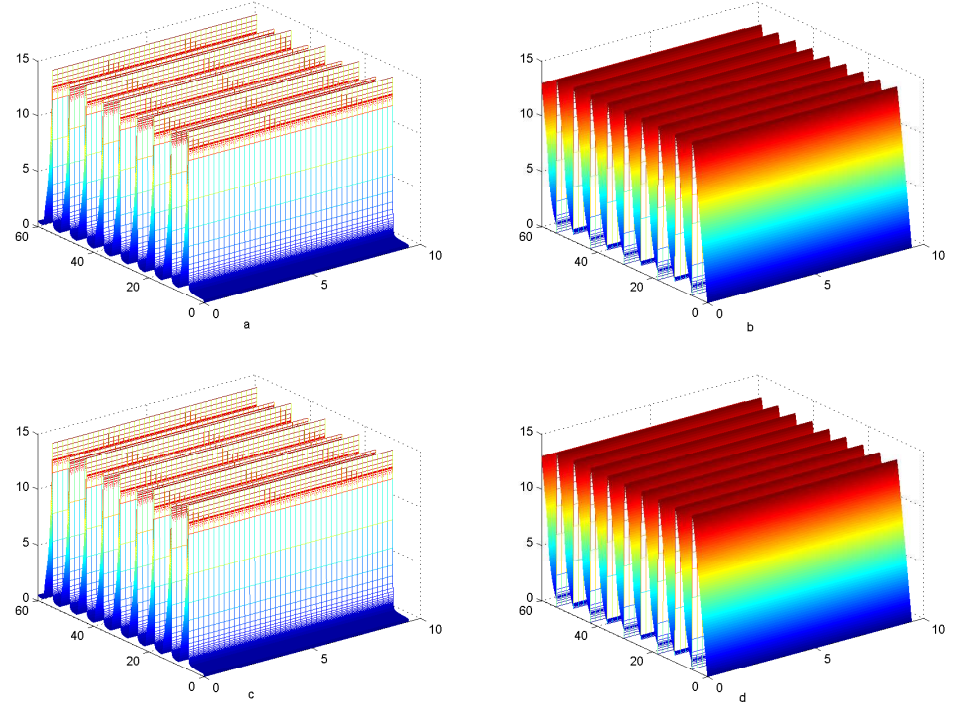

FiguRE 2. Spatially homogenous periodic solutions appear and are asymptotically stable when $b$ crosses through the first Hopf bifurcation value $b=a^{2}+1$, where $a=3, b=11$, and initial data is $(4,2.8,4,2.8)$.

For the spatially non-homogeneous periodic solutions in Theorem 2.2, we have

TheOREM 2.4. For the system (1.2), the Hopf bifurcation at $b=b_{n}^{H} \in \Lambda^{H} \backslash\left\{b_{0,+}^{H}\right\}$ (defined above), is forward (backward) if $\operatorname{Rec}_{1}\left(b_{n}^{H}\right)<0(>0)$, and the bifurcating 
(spatially non-homogeneous) periodic solutions are unstable. Particularly, the bifurcating periodic solutions on the center manifold are orbitally asymptotically stable (unstable) if $\operatorname{Rec}_{1}\left(b_{n}^{H}\right)<0(>0)$.

Proof. The bifurcating periodic solutions are clearly unstable since the characteristic equations $(2.24)$ have positive real-part roots for $b \in\left(a^{2}+1,+\infty\right)$. The calculation of $\operatorname{Rec}_{1}\left(b_{n}^{H}\right)$ is lengthy, and we will give it in Appendix.

\section{Steady state bifurcation}

In this section, by applying the results due to Shi and Wang [22] and Yi, Wei and Shi $[\mathbf{1 8}]$, we shall give the sufficient conditions for the global steady state bifurcation in the general R-D system (2.1). Then, by employing the technique introduced in Zhou and $\mathrm{Mu}$ [17], we investigate the steady state bifurcation for the coupled Brusselator model (1.2).

3.1. Steady state bifurcation for general R-D system. In the following, we consider the general R-D system (2.1) with Neumann boundary condition on spatial domain $\Omega=(0, l \pi)$, where $d_{1}, d_{2}, a, c, l, b$ are positive constants, $f^{i}: R \times$ $R^{m} \rightarrow R, \quad(i=1,2, \cdots, m)$ are $C^{k}(k \geq 2), f^{i}(b, \mathbf{0}) \equiv 0, \quad(i=1,2, \cdots, m)$. $X, L(b), L_{n}(b)$ are defined in Section 2.1, but the domain of linear operators is $X$ not $X_{c}$. Our main assumption is as follows.

(H2) There exists a neighborhood $O$ of $b_{0}$, such that for $b \in O, L(b)$ has a simple real eigenvalue $\gamma(b)$, continuously differentiable in $b$, with $\gamma\left(b_{0}\right)=0$, and $\gamma^{\prime}\left(b_{0}\right) \neq 0$, and all other eigenvalues of $L(b)$ have non-zero real parts for $b \in O$. Let

$$
\mathbf{G}(b, U)=D \triangle U+F(b, U)
$$

From (H2), we can show that $\mathbf{G}$ satisfies the conditions of saddle-node bifurcation theorem of $[\mathbf{2 1}]$. The proof is omitted, (see [18]). Therefore, under the assumption of (H2), saddle-node bifurcation occurs at $b=b_{0}$.

From Shi and Wang [22], we have the following global bifurcation theorem regarding the steady state bifurcation of system (2.1).

THEOREM 3.1. (Global bifurcation theorem) Let I be a closed interval which contains $b_{0} \in R$. Suppose that (H2) holds at $b=b_{0}$. Then there is a smooth curve $\Gamma$ of the steady state solutions of $(2.1)$ bifurcating from $\left(b_{0}, \boldsymbol{O}\right)$, and $\Gamma$ is contained in a connected component $\mathcal{C}$ of the set of non-zero steady state solutions of (2.1) in $I \times X$. Either $\mathcal{C}$ is unbounded in $I \times X$, or $\mathcal{C} \cap(\partial I \times X) \neq \emptyset$, or $\mathcal{C}$ contains a further bifurcation point $\left(b_{*}, \boldsymbol{O}\right)$ with $b_{0} \neq b_{*}$ such that 0 is an eigenvalue of $L\left(b_{*}\right)$. $\Gamma$ can be expressed as $\Gamma=\left\{\left(b(s), u_{1}(s), \cdots, u_{m}(s)\right): s \in(-\epsilon,+\epsilon)\right\}$, where $u_{i}(s)=$ $s a_{i n} \cos \frac{n x}{l}+s \psi_{i}(s),(i=1, \cdots, m)$ for $s \in(-\epsilon,+\epsilon)$, and $b:(-\epsilon,+\epsilon) \rightarrow R, \psi_{i}(s):$ $(-\epsilon,+\epsilon) \rightarrow Z$ are $C^{1}$ functions, such that $b(0)=b_{0}, \psi_{i}(0)=0,(i=1, \cdots, m)$. Here $a_{1 n}, a_{2 n}, \cdots, a_{m n}$ satisfy $L_{n}\left(a_{1 n}, a_{2 n}, \cdots, a_{m n}\right)^{T}=(0,0, \cdots, 0)^{T}$.

3.2. Steady state bifurcation for a coupled Brusselator model. In this subsection, we consider the steady state bifurcation for the coupled Brusselator 
model (1.2). The steady state solutions of (1.2) satisfy the following elliptic system:

$$
\left\{\begin{array}{l}
-d_{1} \triangle u_{1}=c\left(u_{3}-u_{1}\right)+a-(b+1) u_{1}+u_{1}^{2} u_{2}, x \in(0, l \pi), \\
-d_{2} \triangle u_{2}=-b u_{1}-u_{1}^{2} u_{2}, x \in(0, l \pi), \\
-d_{1} \triangle u_{3}=c\left(u_{1}-u_{3}\right)+a-(b+1) u_{3}+u_{3}^{2} u_{4}, x \in(0, l \pi), \\
-d_{2} \triangle u_{4}=-b u_{3}-u_{3}^{2} u_{4}, x \in(0, l \pi), \\
\frac{\partial u_{i}}{\partial x}(0, t)=\frac{\partial u_{i}}{\partial x}(l \pi, t)=0, t>0, i=1,2,3,4 .
\end{array}\right.
$$

System (3.2) has a unique constant equilibrium point $\left(a, \frac{b}{a}, a, \frac{b}{a}\right)$. Now we start to analyze the steady state bifurcation. We also choose $b$ as the main bifurcation parameter with $a, c, d_{1}, d_{2}, l$ fixed and positive. With the same translation as that of Section 2.2, system (3.2) can be reduced to the following system:

$$
\left\{\begin{array}{l}
-d_{1} \triangle u_{1}=(b-c-1) u_{1}+a^{2} u_{2}+c u_{3}+u_{1}^{2} u_{2}+\frac{b}{a} u_{1}^{2}+2 a u_{1} u_{2} \\
-d_{2} \triangle u_{2}=-b u_{1}-a^{2} u_{2}-u_{1}^{2} u_{2}-\frac{b}{a} u_{1}^{2}-2 a u_{1} u_{2} \\
-d_{1} \triangle u_{3}=c u_{1}+(b-c-1) u_{3}+a^{2} u_{4}+u_{3}^{2} u_{4}+\frac{b}{a} u_{3}^{2}+2 a u_{3} u_{4} \\
-d_{2} \triangle u_{4}=-b u_{3}-a^{2} u_{4}-u_{3}^{2} u_{4}-\frac{b}{a} u_{3}^{2}-2 a u_{3} u_{4} \\
\frac{\partial u_{i}}{\partial x}(0, t)=\frac{\partial u_{i}}{\partial x}(l \pi, t)=0, t>0, i=1,2,3,4
\end{array}\right.
$$

The origin $(0,0,0,0)$ is the unique constant equilibrium solution. By the same way as Section 2.2, the characteristic equation of the linearization of $(3.3)$ at $(0,0,0,0)$ is

$$
\left(\lambda^{2}-T_{n}(b) \lambda+D_{n}(b)\right)\left(\lambda^{2}-P_{n}(b) \lambda+Q_{n}(b)\right)=0,
$$

where $T_{n}(b), D_{n}(b), P_{n}(b), Q_{n}(b)$ are defined in Section 2.2. Obviously, $\lambda_{i}$ is the root of (3.4) if and only if $\lambda_{i}$ is the root of the following equations (3.5) or (3.6):

$$
\begin{aligned}
& \lambda^{2}-T_{n}(b) \lambda+D_{n}(b)=0, \\
& \lambda^{2}-P_{n}(b) \lambda+Q_{n}(b)=0 .
\end{aligned}
$$

Now we identify all the possible steady state bifurcation values $b_{0}$, which satisfy the condition (H2). We achieve it in three steps.

Step 1. We shall find out these $b_{0}$, such that at $b=b_{0}$, Eq.(3.5) has a simple zero root, and all other roots of (3.5) have non-zero real parts. That is, there exists $n \in N_{0}=N \cup\{0\}$ such that

$$
D_{n}\left(b_{0}\right)=0, T_{n}\left(b_{0}\right) \neq 0, T_{j}\left(b_{0}\right) \neq 0, D_{j}\left(b_{0}\right) \neq 0 \text { for } \forall j \neq n .
$$

To determine such $b_{0}$, we rewrite

$$
\begin{aligned}
& D_{n}(b)=\left(\frac{d_{2} n^{2}}{l^{2}}+a^{2}\right)\left(\frac{d_{1} n^{2}}{l^{2}}-b+1\right)+b a^{2}, \\
& Q_{n}(b)=\left(\frac{d_{2} n^{2}}{l^{2}}+a^{2}\right)\left(\frac{d_{1} n^{2}}{l^{2}}-b+2 c+1\right)+b a^{2} .
\end{aligned}
$$

Obviously, $D_{0}(b)=a^{2} \neq 0, Q_{0}(b)=a^{2}(2 c+1) \neq 0$, since $a>0$. If $D_{n}(b)=0$, we have $b_{n,+}^{S}=\frac{d_{1} n^{2}}{l^{2}}+\frac{a^{2} l^{2}}{d_{2} n^{2}}+\frac{a^{2} d_{1}}{d_{2}}+1, n=1,2, \cdots$, and $T_{n}\left(b_{n,+}^{S}\right)=\frac{a^{2} l^{2}}{d_{2} n^{2}}-\frac{d_{2} n^{2}}{l^{2}}+$ $\frac{a^{2} d_{1}}{d_{2}}-a^{2}, n=1,2, \cdots$.

To let $T_{n}\left(b_{n,+}^{S}\right) \neq 0$, we need to prove the following inequality.

$$
d_{2}\left(\frac{n^{2}}{l^{2}}\right)^{2}+a^{2}\left(1-\frac{d_{1}}{d_{2}}\right) \frac{n^{2}}{l^{2}}-\frac{a^{2}}{d_{2}} \neq 0,
$$


or equivalently,

$$
n^{2} \neq \frac{l^{2}}{2 d_{2}^{2}}\left[\left(d_{1}-d_{2}\right) a^{2}+\sqrt{\left(d_{2}-d_{1}\right)^{2} a^{4}+4 d_{2}^{2} a^{2}}\right] .
$$

To let $D_{m}\left(b_{n,+}^{S}\right) \neq 0$, for $\forall m \neq n$, we have

$$
m n \neq \frac{a l^{2}}{\sqrt{d_{1} d_{2}}}, \forall m \neq n .
$$

To let $T_{j}\left(b_{n,+}^{S}\right) \neq 0$, for $\forall j \neq n$, we have

$$
\frac{d_{1} n^{2}}{l^{2}}+\frac{a^{2} l^{2}}{d_{2} n^{2}}+\frac{a^{2} d_{1}}{d_{2}} \neq \frac{\left(d_{1}+d_{2}\right) j^{2}}{l^{2}}+a^{2}, \forall j \neq n .
$$

In this case, all the possible parameters $b_{0}$ can be expressed as

$\Lambda_{1}^{S}:=\left\{b_{n,+}^{S}=\frac{d_{1} n^{2}}{l^{2}}+\frac{a^{2} l^{2}}{d_{2} n^{2}}+\frac{a^{2} d_{1}}{d_{2}}+1, n=1,2, \cdots\right.$, and $n$ satisfies $\left.(3.9)-(3.11)\right\}$.

Step 2. We shall find out these $b_{0}$, such that at $b=b_{0}$, Eq.(3.6) has a simple zero root, and all other roots of (3.6) have non-zero real parts.

Similarly, if $Q_{n}(b)=0$, we have $b_{n,-}^{S}=\frac{d_{1} n^{2}}{l^{2}}+(2 c+1) \frac{a^{2} l^{2}}{d_{2} n^{2}}+\left(2 c+1+\frac{a^{2} d_{1}}{d_{2}}\right), n=$ $1,2, \cdots$. To let $P_{n}\left(b_{n,-}^{S}\right) \neq 0$, we have

$$
n^{2} \neq \frac{l^{2}}{2 d_{2}^{2}}\left[\left(d_{1}-d_{2}\right) a^{2}+\sqrt{\left(d_{2}-d_{1}\right)^{2} a^{4}+4 d_{2}^{2} a^{2}(2 c+1)}\right] .
$$

To let $Q_{m}\left(b_{n,-}^{S}\right) \neq 0$, for $\forall m \neq n$, we have

$$
m n \neq a l^{2} \sqrt{\frac{2 c+1}{d_{1} d_{2}}}, \forall m \neq n .
$$

To let $P_{j}\left(b_{n,-}^{S}\right) \neq 0$, for $\forall j \neq n$, we have

$$
\frac{d_{1} n^{2}}{l^{2}}+\frac{a^{2} l^{2}(2 c+1)}{d_{2} n^{2}}+\frac{a^{2} d_{1}}{d_{2}} \neq \frac{\left(d_{1}+d_{2}\right) j^{2}}{l^{2}}+a^{2}, \text { for } \forall j \neq n .
$$

In this case, such $b_{0}$ can be labeled as

$$
\Lambda_{2}^{S}:=\left\{b_{n,-}^{S}, n=1,2, \cdots, \text { and } n \text { satisfies }(3.13)-(3.15)\right\} .
$$

Step 3. The transversality condition holds.

From (3.8), it follows that

$$
\left.\frac{d D_{n}(b)}{d b}\right|_{b=b_{n,+}^{S}}=-\frac{d_{2} n^{2}}{l^{2}} \neq 0, \quad(n=1,2, \cdots),
$$

and

$$
\left.\frac{d Q_{n}(b)}{d b}\right|_{b=b_{n,-}^{S}}=-\frac{d_{2} n^{2}}{l^{2}} \neq 0, \quad(n=1,2, \cdots) .
$$

For convenience, we denote

$$
\Lambda^{S}=\Lambda_{1}^{S} \cup \Lambda_{2}^{S} \triangleq\left\{b_{n}^{S}\right\} .
$$

Since $b_{n,+}^{S} \rightarrow+\infty$ and $b_{n,-}^{S} \rightarrow+\infty$, as $n \rightarrow \infty$, the sequences $\left\{b_{n,+}^{S}\right\}$ and $\left\{b_{n,-}^{S}\right\}$ have no accumulation points. Therefore, $\Lambda^{S}=\left\{b_{n}^{S}\right\}$ has no accumulation points and can be sequenced as

$$
b_{1}^{S}<b_{2}^{S}<\cdots<b_{n}^{S}<\cdots \rightarrow+\infty .
$$


From the above analysis, we know that the local steady state bifurcation occurs at $b=b_{n}^{S} \in \Lambda^{S}$.

In order to consider global steady state bifurcation, we recall some prior estimates for every solution of system (3.2). We make a simple scaling to system (3.2) as follows,

$$
d_{1}^{\prime}=d_{1}, d_{2}^{\prime}=\frac{d_{2}}{a^{2}}, u=\frac{u_{1}}{a}, v=\frac{a u_{2}}{b}, w=\frac{u_{3}}{a}, z=\frac{a u_{4}}{b},
$$

then (3.2) becomes

$$
\left\{\begin{array}{l}
-d_{1}^{\prime} \triangle u=1-(b+1) u+b u^{2} v+c(w-u), x \in(0, l \pi) \\
-d_{2}^{\prime} \triangle v=u-u^{2} v, x \in(0, l \pi) \\
-d_{1}^{\prime} \triangle w=1-(b+1) w+b w^{2} z+c(u-w), x \in(0, l \pi) \\
-d_{2}^{\prime} \triangle z=w-w^{2} z, x \in(0, l \pi) \\
\frac{\partial u}{\partial x}(0, t)=\frac{\partial v}{\partial x}(0, t)=\frac{\partial w}{\partial x}(0, t)=\frac{\partial z}{\partial x}(0, t)=0 \\
\frac{\partial u}{\partial x}(l \pi, t)=\frac{\partial v}{\partial x}(l \pi, t)=\frac{\partial w}{\partial x}(l \pi, t)=\frac{\partial z}{\partial x}(l \pi, t)=0
\end{array}\right.
$$

which is the system (4) of Zhou and Mu [17]. Applying the Corollary 3 of [17]), we have the following prior estimates.

Lemma 3.2. Let $B, D, D_{1}$ and $D_{2}$ be given positive numbers. Then there exists a positive constant $K^{\prime}$, which depends on $B, D, D_{1}, D_{2}$ and $\Omega$, such that if $0<b \leq$ $B, d_{1}^{\prime} \geq D$ and $D_{1} \leq d_{2}^{\prime} \leq D_{2}$, any solution $(u, v, w, z)$ of system (3.22) satisfies

$$
\|u\|_{1,2}+\|v\|_{1,2}+\|w\|_{1,2}+\|z\|_{1,2} \leq K^{\prime} .
$$

Then it follows from the change of variables (3.21) that

$$
\begin{aligned}
& \left\|u_{1}\right\|_{1,2}+\left\|u_{2}\right\|_{1,2}+\left\|u_{3}\right\|_{1,2}+\left\|u_{4}\right\|_{1,2} \\
& \leq a\|u\|_{1,2}+\frac{b}{a}\|v\|_{1,2}+a\|w\|_{1,2}+\frac{b}{a}\|z\|_{1,2} \\
& \leq \max \left\{a, \frac{b}{a}\right\}\left[\|u\|_{1,2}+\|v\|_{1,2}+\|w\|_{1,2}+\|z\|_{1,2}\right] \\
& \leq \max \left\{a, \frac{b}{a}\right\} K^{\prime} \triangleq K .
\end{aligned}
$$

Consequently, we have the following prior estimates.

LEMMA 3.3. Let $B, d_{1}, d_{2}, a, c, l$ be given positive numbers, there exists a positive constant $K$, which depends only on $B, d_{1}, d_{2}, a, c, l$ such that if $0<b<B$, any solution $\left(u_{1}, u_{2}, u_{3}, u_{4}\right)$ of system (3.2), satisfies

$$
\left\|u_{1}\right\|_{1,2}+\left\|u_{2}\right\|_{1,2}+\left\|u_{3}\right\|_{1,2}+\left\|u_{4}\right\|_{1,2} \leq K
$$

We claim that there are only countably many $l>0$, such that $b_{i,+}^{S}=b_{j,+}^{S},(i \neq$ $j)$, or $b_{i,+}^{S}=b_{j,-}^{S}$ or $b_{i,-}^{S}=b_{j,-}^{S},(i \neq j)$ or $b_{i,+}^{S}=b_{j,+}^{H}$ or $b_{i,+}^{H}=b_{j,-}^{H}$ or $b_{i,-}^{S}=b_{j,+}^{H}$ or $b_{i,+}^{S}=b_{j,-}^{H}$ for these $l$ and some $i, j \in N$.

In fact, $b_{i,+}^{S}=b_{j,+}^{S},(i \neq j)$ is equivalent to $l^{4}=\frac{d_{1} d_{2}}{a^{2}} i^{2} j^{2}$, for some $i, j \in N$. Therefore, $l$ is at most countable since $i, j$ are at most countable. Other equalities are discussed similarly. We define

$$
\begin{gathered}
L^{E}=\left\{l>0, b_{i,+}^{S}=b_{j,+}^{S},(i \neq j) \text { or } b_{i,+}^{S}=b_{j,-}^{S} \text { or } b_{i,-}^{S}=b_{j,-}^{S},(i \neq j) \text { or } b_{i,+}^{S}=b_{j,+}^{H}\right. \\
\text { or } \left.b_{i,+}^{H}=b_{j,-}^{H} \text { or } b_{i,-}^{S}=b_{j,+}^{H} \text { or } b_{i,+}^{S}=b_{j,-}^{H} \text { for some } i, j \in N\right\} .
\end{gathered}
$$


Then the points in $L^{E}$ can be arranged as a sequence whose only limit point is $\infty$.

By Theorem 1.2 of Zhou and $\mathrm{Mu}[\mathbf{1 7}]$, we know that, there exists $b_{0}>0$, such that the system (3.22) has no non-constant solutions for $b \in\left(0, b_{0}\right)$. Then for system (3.2), we have the following results.

Lemma 3.4. Let $d_{1}, d_{2}$ be fixed. Then there exists $b_{0}>0$, which depends only on $d_{1}, d_{2}$ and $\Omega$, such that (3.2) has no non-constant solution provided that $0<$ $b \leq b_{0}$, i.e. the only solution for (3.2) is $(u, v, w, z)=\left(a, \frac{b}{a}, a, \frac{b}{a}\right)$.

Summarizing the preparation above, we are now ready to state the main results of this subsection on the global bifurcation of steady state solutions of (3.2).

THEOREM 3.5. Suppose that $d_{1}, d_{2}, a, c$ and $l$ are positive constants. Then for $l \notin L^{E},\left\{b_{n}^{S}\right\}$ (defined above) is a sequence of steady state bifurcation values of system (3.2). Moreover, there is a smooth curve $\Gamma_{n}$ of positive solutions of (3.2) bifurcating from $\left(b_{n}^{S}, a, \frac{b_{n}^{S}}{a}, a, \frac{b_{n}^{S}}{a}\right)$, with $\Gamma_{n}$ contained in a global branch $\mathcal{C}_{n}$ of the positive solutions of (3.2). Furthermore,

1. Near $\left(b_{n}^{S}, a, \frac{b_{n}^{S}}{a}, a, \frac{b_{n}^{S}}{a}\right), \Gamma_{n}=\left\{\left(b(s), u_{1}(s), u_{2}(s), u_{3}(s), u_{4}(s)\right): s \in(-\epsilon,+\epsilon)\right\}$, where

$$
\begin{aligned}
& u_{1}(s)=a+s a_{n} \cos \frac{n x}{l}+s \psi_{1}(s), u_{2}(s)=\frac{b_{n}^{S}}{a}+s b_{n} \cos \frac{n x}{l}+s \psi_{2}(s), \\
& u_{3}(s)=a+s c_{n} \cos \frac{n x}{l}+s \psi_{3}(s), u_{4}(s)=\frac{b_{n}^{S}}{a}+s d_{n} \cos \frac{n x}{l}+s \psi_{4}(s),
\end{aligned}
$$

for $s \in(-\epsilon,+\epsilon)$ for some $C^{\infty}$ smooth functions. $b, \psi_{i}(i=1,2,3,4)$ such that $b(0)=b_{n}^{S}$ and $\psi_{i}(0)=0(i=1,2,3,4)$. Here $a_{n}, b_{n}, c_{n}$ and $d_{n}$ satisfy

$$
L_{n}\left(b_{n}^{S}\right)\left(a_{n}, b_{n}, c_{n}, d_{n}\right)^{T}=(0,0,0,0)^{T} \text {. }
$$

2. Either $\mathcal{C}_{n}$ contains another bifurcation point $\left(b_{j}^{S}, a, \frac{b_{j}^{S}}{a}, a, \frac{b_{j}^{S}}{a}\right)$ with $b_{j}^{S} \neq b_{n}^{S}$, or the projection of $\mathcal{C}_{n}$ onto b-axis contains the interval $\left(b_{n}^{S},+\infty\right)$.

Proof. From the discussion above, the conditions in Theorem 3.1 are satisfied at $b=b_{n}^{S} \in \Lambda^{S}$. We can apply the global bifurcation theorem 3.1, and confine the interval $b \in\left(b_{0},+\infty\right)$, ( $b_{0}$ is given in Lemma 3.4). From Lemma 3.3, all solutions of (3.2) are uniformly bounded in $X$ if $b \in\left(b_{0},+\infty\right)$. Hence, the global branch $\mathcal{C}_{n}$ is bounded in $X$ if $b \in\left(b_{0},+\infty\right)$. From Lemma 3.4, there are no non-constant solution of $(3.2)$ if $b \in\left(0, b_{0}\right]$. Hence $\mathcal{C}_{n}$ can't intersect the boundary $\left\{b=b_{0}\right\} \times X$. Therefore, the conclusion is derived.

Finally, we discuss the interaction between the Hopf and steady state bifurcations. For a countably set of values $l \in L^{E}, b_{j,+}^{H}$ and $b_{i,-}^{H}$, or $b_{i,+}^{S}$ and $b_{j,-}^{S}$, or $b_{i}^{H}$ and $b_{j}^{S}$ can be identical for some $i, j$, so that system (3.2) has a higher-dimensional center manifold near $\left(a, \frac{b}{a}, a, \frac{b}{a}\right)$ at such $b$. Bifurcations from these points with higher-dimensional degeneracy are still possible, but we don't consider them here. For other $l \notin L^{E}$, we have shown that in some necessary conditions, Hopf bifurcations and steady state bifurcations could occur at these points (see Theorems 2.2, $2.3,3.5)$. In fact, the occurrence of Hopf bifurcation and steady state bifurcation depends only on the specific eigen-mode $\cos \frac{n x}{l}$, the bifurcation related to this mode has the following possible scenarios: 
Case 1. Both of $b_{n,+}^{H}, b_{j,-}^{H}\left(n=0,1, \cdots, N ; j=0,1, \cdots, N^{\prime}\right)$ and $b_{n, \pm}^{S}$ exist, then there are two steady state bifurcations and two Hopf bifurcations for this mode;

Case 2. $b_{n, \pm}^{S}$ exist but not $b_{n,+}^{H}$ or $b_{j,-}^{H}\left(n=0,1, \cdots, N ; j=0,1, \cdots, N^{\prime}\right)$, then there are two steady state bifurcations and no Hopf bifurcations for this mode;

Case $3 . b_{n, \pm}^{S}$ and $b_{j,-}^{H}\left(j=0,1, \cdots, N^{\prime}\right)$ exist but not $b_{n,+}^{H}(n=0,1, \cdots, N)$, then there are two steady state bifurcations and one Hopf bifurcation for this mode;

Case $4 . b_{n,+}^{H}$ and $b_{j,+}^{H}$ exist but not $b_{n}^{S}$, then there are two Hopf bifurcation points and no steady state bifurcation points.

Example 3.1. In Figure 3, for $1 \leq n \leq 12$ and $n \in N$, Case 1 occurs, and there exist 24 Hopf bifurcation points and 24 steady state bifurcation points. For $n=13$, Case 3 occurs, and there exist 1 Hopf bifurcation point and 2 steady state bifurcation points. For $n=0$, Case 4 occurs, and there exist 2 Hopf bifurcations and no steady state bifurcations. For $n \geq 14$, Case 2 occurs, and there exist $2(n-13)$ steady state bifurcation points and no Hopf bifurcation points.



FiguRE 3. Graph of $T_{n}(b, p) P_{n} \stackrel{\mathrm{b}}{(b, p)}=0$ and $D_{n}(b, p) Q_{n}(b, p)=0$. Here $d_{1}=3, d_{2}=1, a=3, c=3$. The horizontal lines are $p=\frac{n^{2}}{l^{2}}$, where $n \in\{0,1,2, \cdots\}$. 
Appendix. Bifurcation direction of the spatially non-homogeneous periodic solutions

In the appendix, we determine the bifurcation direction of spatially non-homogeneous periodic solutions and stability of the bifurcation periodic solutions on the center manifold. Recall that the bifurcating periodic solution is forward (backward) if $\left.\frac{\operatorname{Re}\left(c_{1}(b)\right)}{\alpha^{\prime}(b)}\right|_{b=b_{n}^{H}}<0(>0)$. Since

$$
\left.\alpha_{1}^{\prime}(b)\right|_{b=b_{n,+}^{H}}>0(n=1, \cdots, N),\left.\alpha_{2}^{\prime}(b)\right|_{b=b_{n,-}^{H^{\prime}}}>0\left(n=0, \cdots, N^{\prime}\right),
$$

we only need to calculate $\operatorname{Rec}_{1}\left(b_{n}^{H}\right)$. Since $\left\{b_{n}^{H}\right\}=\left\{b_{n,+}^{H}\right\} \cup\left\{b_{n,-}^{H}\right\}$, for convenience, we only calculate $\operatorname{Rec}_{1}\left(b_{n,+}^{H}\right)$. When $b=b_{n,+}^{H}, n=1,2, \cdots, N$, we set

$$
\begin{aligned}
q & :=\cos \frac{n}{l} x\left(a_{1 n}, a_{2 n}, a_{3 n}, a_{4 n}\right)^{T} \\
& =\cos \frac{n}{l} x\left(-\frac{1}{b_{n,+}^{H}}\left(\frac{d_{2} n^{2}}{l^{2}}+a^{2}+i \omega_{n}\right), 1,-\frac{1}{b_{n,+}^{H}}\left(\frac{d_{2} n^{2}}{l^{2}}+a^{2}+i \omega_{n}\right), 1\right)^{T}, \\
q^{*^{\prime}} & :=\cos \frac{n}{l} x\left(a_{1 n}^{*}, a_{2 n}^{*}, a_{3 n}^{*}, a_{4 n}^{*}\right)^{T} \\
& =\cos \frac{n}{l} x\left(\frac{1}{a^{2}}\left(\frac{d_{2} n^{2}}{l^{2}}+a^{2}-i \omega_{n}\right), 1, \frac{1}{a^{2}}\left(\frac{d_{2} n^{2}}{l^{2}}+a^{2}-i \omega_{n}\right), 1\right)^{T},
\end{aligned}
$$

where $b_{n,+}^{H}=\frac{\left(d_{1}+d_{2}\right) n^{2}}{l^{2}}+a^{2}+1, \omega_{n}=\sqrt{-d_{2}^{2}\left(\frac{n^{2}}{l^{2}}\right)^{2}+a^{2}\left(d_{1}-d_{2}\right) \frac{n^{2}}{l^{2}}+a^{2}}$.

Let

$$
q^{*}=D q^{*^{\prime}}=D \cos \frac{n}{l} x\left(\frac{1}{a^{2}}\left(\frac{d_{2} n^{2}}{l^{2}}+a^{2}-i \omega_{n}\right), 1, \frac{1}{a^{2}}\left(\frac{d_{2} n^{2}}{l^{2}}+a^{2}-i \omega_{n}\right), 1\right)^{T},
$$

where $\bar{D}=\left\{\left[1-\frac{1}{a^{2} b_{n,+}^{H}}\left(\frac{d_{2} n^{2}}{l^{2}}+a^{2}+i \omega_{n}\right)^{2}\right] l \pi\right\}^{-1}$. By calculation, we know that $q$ and $q^{*}$ satisfy the following conditions:

$$
L\left(b_{n,+}^{H}\right) q=i \omega_{n} q, L^{*}\left(b_{n,+}^{H}\right) q^{*}=-i \omega_{n} q^{*},\left\langle q^{*}, q\right\rangle=1 .
$$

By (2.19), when $n \in N$, it follows that

$$
\left\langle q^{*}, Q_{q q}\right\rangle=\left\langle q^{*}, Q_{q \bar{q}}\right\rangle=0 .
$$

Thus, in order to calculate $\operatorname{Rec}_{1}\left(b_{n,+}^{H}\right)$, it remains to calculate

$$
\left\langle q^{*}, Q_{W_{11} q}\right\rangle,\left\langle q^{*}, Q_{W_{20} \bar{q}}\right\rangle \text {, and }\left\langle q^{*}, C_{q q \bar{q}}\right\rangle \text {. }
$$

It is straightforward to compute that

$$
\left[2 i \omega_{n} I-L_{2 n}\left(b_{n,+}^{H}\right)\right]^{-1}=\left[\begin{array}{cc}
A_{1} & B_{1} \\
B_{1} & A_{1}
\end{array}\right]
$$

where

$$
A_{1}=\left[\begin{array}{cc}
\frac{E}{E^{2}-c^{2}} & \frac{E a^{2}}{\left(E^{2}-c^{2}\right)\left(2 i \omega_{n}+\frac{4 d_{2} n^{2}}{l^{2}}+a^{2}\right)} \\
\frac{-E b_{n,+}^{H}}{\left.E^{2}-c^{2}\right)\left(2 i \omega_{n}+\frac{4 d_{2} n^{2}}{l^{2}}+a^{2}\right)} & \frac{\left(E^{2}-c^{2}\right)\left(2 i \omega_{n}+\frac{4 d_{2} n^{2}}{l^{2}}+a^{2}\right)-E a^{2} b_{n,+}^{H}}{\left(E^{2}-c^{2}\right)\left(2 i \omega_{n}+\frac{4 d_{2} n^{2}}{l^{2}}+a^{2}\right)^{2}}
\end{array}\right],
$$

and

$$
B_{1}=\left[\begin{array}{cc}
\frac{c}{E^{2}-c^{2}} & \frac{a^{2} c}{\left(E^{2}-c^{2}\right)\left(2 i \omega_{n}+\frac{4 d_{2} n^{2}}{l^{2}}+a^{2}\right)} \\
\frac{-c b_{n,+}^{H}}{\left(E^{2}-c^{2}\right)\left(2 i \omega_{n}+\frac{4 d_{2} n^{2}}{l^{2}}+a^{2}\right)} & \frac{-a^{2} c b_{n,+}^{H}}{\left(E^{2}-c^{2}\right)\left(2 i \omega_{n}+\frac{4 d_{2} n^{2}}{l^{2}}+a^{2}\right)^{2}}
\end{array}\right],
$$




$$
E=\frac{a^{2} b_{n,+}^{H}}{2 i \omega_{n}+\frac{4 d_{2} n^{2}}{l^{2}}+a^{2}}+2 i \omega_{n}+\frac{4 d_{1} n^{2}}{l^{2}}-b_{n,+}^{H}+c+1,
$$

and

$$
\left[2 i \omega_{n} I-L_{0}\left(b_{n,+}^{H}\right)\right]^{-1}=\left[\begin{array}{cc}
A_{2} & B_{2} \\
B_{2} & A_{2}
\end{array}\right]
$$

with

and

$$
A_{2}=\left[\begin{array}{cc}
\frac{F}{F^{2}-c^{2}} & \frac{F a^{2}}{\left(F^{2}-c^{2}\right)\left(2 i \omega_{n}+a^{2}\right)} \\
\frac{-F b_{n,+}^{H}}{\left(F^{2}-c^{2}\right)\left(2 i \omega_{n}+a^{2}\right)} & \frac{\left(F^{2}-c^{2}\right)\left(2 i \omega_{n}+a^{2}\right)-F a^{2} b_{n,+}^{H}}{\left(F^{2}-c^{2}\right)\left(2 i \omega_{n}+a^{2}\right)^{2}}
\end{array}\right]
$$

$$
\begin{gathered}
B_{2}=\left[\begin{array}{cc}
\frac{c}{F^{2}-c^{2}} & \frac{a^{2} c}{\left(F^{2}-c^{2}\right)\left(2 i \omega_{n}+a^{2}\right)} \\
\frac{-c b_{n,+}^{H}}{\left(F^{2}-c^{2}\right)\left(2 i \omega_{n}+a^{2}\right)} & \frac{-a^{2} c b_{n,+}^{H}}{\left(F^{2}-c^{2}\right)\left(2 i \omega_{n}+a^{2}\right)^{2}}
\end{array}\right], \\
F=\frac{a^{2} b_{n,+}^{H}}{2 i \omega_{n}+a^{2}}+2 i \omega_{n}-b_{n,+}^{H}+c+1 .
\end{gathered}
$$

Then, we have by (2.20), when $n \in N$,

$$
\begin{aligned}
W_{20} & =\left[\frac{\left[2 i \omega_{n} I-L_{2 n}\left(b_{n,+}^{H}\right)\right]^{-1}}{2} \cos \frac{2 n}{l} x+\frac{\left[2 i \omega_{n} I-L_{0}\left(b_{n,+}^{H}\right)\right]^{-1}}{2}\right]\left(b_{1 n}, b_{2 n}, b_{3 n}, b_{4 n}\right)^{T} \\
& =(\xi, \eta, \xi, \eta)^{T} \cos \frac{2 n}{l} x+(\tilde{\xi}, \tilde{\eta}, \tilde{\xi}, \tilde{\eta})^{T}
\end{aligned}
$$

where

$$
\begin{gathered}
\xi=\frac{4 d_{2} n^{2}+2 i \omega_{n} l^{2}}{(E-c)\left(4 d_{2} n^{2}+a^{2} l^{2}+2 i \omega_{n} l^{2}\right)} b_{1 n} \\
\eta=\left[\frac{-\left(4 d_{2} n^{2}+2 i \omega_{n} l^{2}\right) b_{n,+}^{H} l^{2}}{(E-c)\left(4 d_{2} n^{2}+a^{2} l^{2}+2 i \omega_{n} l^{2}\right)^{2}}-\frac{l^{2}}{4 d_{2} n^{2}+a^{2} l^{2}+2 i \omega_{n} l^{2}}\right] b_{1 n} \\
\tilde{\xi}=\frac{2 i \omega_{n}}{(F-c)\left(a^{2}+2 i \omega_{n}\right)} b_{1 n} \\
\tilde{\eta}=\left[\frac{-2 i \omega_{n} b_{n,+}^{H}}{(F-c)\left(a^{2}+2 i \omega_{n}\right)^{2}}-\frac{1}{a^{2}+2 i \omega_{n}}\right] b_{1 n} .
\end{gathered}
$$

Likewise, when $n \in N$,

$$
\begin{aligned}
W_{11} & =-\left[L_{2 n}^{-1}\left(b_{n,+}^{H}\right) \cos \frac{2 n}{l} x+L_{0}^{-1}\left(b_{n,+}^{H}\right)\right]\left(c_{1 n}, c_{2 n}, c_{3 n}, c_{4 n}\right)^{T} \\
& =(\tau, \chi, \tau, \chi)^{T} \cos \frac{2 n}{l} x+(\tilde{\tau}, \tilde{\chi}, \tilde{\tau}, \tilde{\chi})^{T}
\end{aligned}
$$

where

$$
\begin{aligned}
\tau & =\frac{4 d_{2} n^{2}}{(M+c)\left(4 d_{2} n^{2}+a^{2} l^{2}\right)} c_{1 n}, \\
\chi & =\left[\frac{-4 d_{2} b_{n,+}^{H} n^{2} l^{2}}{(M+c)\left(4 d_{2} n^{2}+a^{2} l^{2}\right)^{2}}+\frac{l^{2}}{4 d_{2} n^{2}+a^{2} l^{2}}\right] c_{1 n}, \\
\tilde{\tau} & =0, \tilde{\chi}=\frac{1}{a^{2}} c_{1 n}, \\
M & =\frac{-a^{2} b_{n,+}^{H}}{\frac{4 d_{2} n^{2}}{l^{2}}+a^{2}}-\frac{4 d_{1} n^{2}}{l^{2}}+b_{n,+}^{H}-c-1 .
\end{aligned}
$$


Recall $f^{(1)}\left(b, u_{1}, u_{2}, u_{3}, u_{4}\right)=u_{1}^{2} u_{2}+\frac{b}{a} u_{1}^{2}+2 a u_{1} u_{2}=-f^{(2)}\left(b, u_{1}, u_{2}, u_{3}, u_{4}\right)$,

$$
f^{(3)}\left(b, u_{1}, u_{2}, u_{3}, u_{4}\right)=u_{3}^{2} u_{4}+\frac{b}{a} u_{3}^{2}+2 a u_{3} u_{4}=-f^{(4)}\left(b, u_{1}, u_{2}, u_{3}, u_{4}\right) .
$$

By Section 2.1, we can obtain

$$
\begin{aligned}
b_{1 n} & =-b_{2 n}=b_{3 n}=-b_{4 n}=\frac{2 b_{n,+}^{H}}{a} a_{1 n}^{2}+4 a a_{1 n} a_{2 n} \\
& =\frac{2}{a b_{n,+}^{H}}\left(\frac{d_{2}^{2} n^{4}}{l^{4}}-\omega_{n}^{2}-a^{4}+\frac{2 d_{2} \omega_{n}}{l^{2}} n^{2} i\right) \\
c_{1 n} & =-c_{2 n}=c_{3 n}=-c_{4 n}=\frac{2 b_{n,+}^{H}}{a}\left|a_{1 n}\right|^{2}+2 a\left(a_{1 n} \bar{a}_{2 n}+\bar{a}_{1 n} a_{2 n}\right) \\
& =2 a-\frac{4 a}{b_{n,+}^{H}}\left(a^{2}+\frac{d_{2} n^{2}}{l^{2}}\right), \\
d_{1 n} & =-d_{2 n}=d_{3 n}=-d_{4 n}=2\left(a_{1 n}^{2} \bar{a}_{2 n}+2\left|a_{1 n}\right|^{2} a_{2 n}\right) \\
& =\frac{2}{\left(b_{n,+}^{H}\right)^{2}}\left(a^{2}+\frac{d_{2} n^{2}}{l^{2}}+i \omega_{n}\right)^{2}+\frac{4 a^{2}}{b_{n,+}^{H}} .
\end{aligned}
$$

Here and in the following we always assume that all the partial derivatives of $f^{(i)}(i=1,2,3,4)$ are evaluated at $\left(b_{n,+}^{H}, 0,0,0,0\right)$. Then we have

$$
\begin{aligned}
Q_{W_{20} \bar{q}} & =(1,-1,1,-1)^{T} Q_{W_{20} \bar{q}}^{(1)}, \\
Q_{W_{11} q} & =(1,-1,1,-1)^{T} Q_{W_{11} q}^{(1)}, \\
C_{q q \bar{q}} & =(1,-1,1,-1)^{T} d_{1 n} \cos ^{3} \frac{n x}{l},
\end{aligned}
$$

where

$$
\begin{aligned}
Q_{W_{20} \bar{q}}^{(1)}= & f_{u_{1} u_{1}}^{(1)}\left(\xi \cos \frac{2 n}{l} x+\tilde{\xi}\right) \bar{a}_{1 n} \cos \frac{n}{l} x \\
& +f_{u_{1} u_{2}}^{(1)}\left[\left(\xi \cos \frac{2 n}{l} x+\tilde{\xi}\right) \cos \frac{n}{l} x+\left(\eta \cos \frac{2 n}{l} x+\tilde{\eta}\right) \bar{a}_{1 n} \cos \frac{n}{l} x\right] \\
= & \left(\frac{2 b_{n,+}^{H}}{a} \xi \bar{a}_{1 n}+2 a \xi+2 a \eta \bar{a}_{1 n}\right) \cos \frac{2 n}{l} x \cos \frac{n}{l} x \\
& +\left(\frac{2 b_{n,+}^{H}}{a} \tilde{\xi} \bar{a}_{1 n}+2 a \tilde{\xi}+2 a \tilde{\eta} \bar{a}_{1 n}\right) \cos \frac{n}{l} x . \\
Q_{W_{11} q}^{(1)}= & f_{u_{1} u_{1}}^{(1)}\left(\tau \cos \frac{2 n}{l} x+\tilde{\tau}\right) a_{1 n} \cos \frac{n}{l} x \\
& +f_{u_{1} u_{2}}^{(1)}\left[\left(\tau \cos \frac{2 n}{l} x+\tilde{\tau}\right) \cos \frac{n}{l} x+\left(\chi \cos \frac{2 n}{l} x+\tilde{\chi}\right) a_{1 n} \cos \frac{n}{l} x\right] \\
= & \left(\frac{2 b_{n,+}^{H}}{a} \tau a_{1 n}+2 a \tau+2 a \chi a_{1 n}\right) \cos \frac{2 n}{l} x \cos \frac{n}{l} x \\
& +\left(\frac{2 b_{n,+}^{H}}{a} \tilde{\tau} a_{1 n}+2 a \tilde{\tau}+2 a \tilde{\chi} a_{1 n}\right) \cos \frac{n}{l} x .
\end{aligned}
$$

Notice that for any $n \in N$,

$$
\int_{0}^{l \pi} \cos ^{2} \frac{n}{l} x d x=\frac{l \pi}{2}, \int_{0}^{l \pi} \cos \frac{2 n}{l} x \cos ^{2} \frac{n}{l} x d x=\frac{l \pi}{4}, \int_{0}^{l \pi} \cos ^{4} \frac{n}{l} x d x=\frac{3}{8} l \pi .
$$


We have

$$
\begin{aligned}
\left\langle q^{*}, Q_{W_{20} \bar{q}}\right\rangle= & \left(\bar{a}_{1 n}^{*}-\bar{a}_{2 n}^{*}+\bar{a}_{3 n}^{*}-\bar{a}_{4 n}^{*}\right) \int_{0}^{l \pi} Q_{W_{20} \bar{q}}^{(1)} d x \\
= & \frac{l \pi}{2 a^{2}}\left(\frac{d_{2} n^{2}}{l^{2}}+i \omega_{n}\right)\left(\frac{2 b_{n,+}^{H}}{a} \xi \bar{a}_{1 n}+2 a \xi+2 a \eta \bar{a}_{1 n}\right) \\
& +\frac{l \pi}{a^{2}}\left(\frac{d_{2} n^{2}}{l^{2}}+i \omega_{n}\right)\left(\frac{2 b_{n,+}^{H}}{a} \tilde{\xi} \bar{a}_{1 n}+2 a \tilde{\xi}+2 a \tilde{\eta} \bar{a}_{1 n}\right), \\
\left\langle q^{*}, Q_{W_{11} q}\right\rangle= & \frac{l \pi}{2 a^{2}}\left(\frac{d_{2} n^{2}}{l^{2}}+i \omega_{n}\right)\left(\frac{2 b_{n,+}^{H}}{a} \tau a_{1 n}+2 a \tau+2 a \chi a_{1 n}\right) \\
& +\frac{l \pi}{a^{2}}\left(\frac{d_{2} n^{2}}{l^{2}}+i \omega_{n}\right)\left(\frac{2 b_{n,+}^{H}}{a} \tilde{\tau} a_{1 n}+2 a \tilde{\tau}+2 a \tilde{\chi} a_{1 n}\right), \\
\left\langle q^{*}, C_{q q \bar{q}}\right\rangle= & \frac{3}{4} l \pi\left(\frac{d_{2} n^{2}}{l^{2}}+i \omega_{n}\right) d_{1 n},
\end{aligned}
$$

and

$$
\begin{aligned}
\operatorname{Rec}_{1}\left(b_{n}^{H}\right) & =\operatorname{Re}\left\{\frac{i}{2 \omega_{n}}\left(g_{20} g_{11}-2\left|g_{11}\right|^{2}-\frac{1}{3}\left|g_{02}\right|^{2}\right)\right\}+\operatorname{Re} \frac{g_{21}}{2} \\
& =\operatorname{Re}\left\langle q^{*}, Q_{W_{11} q}\right\rangle+\frac{1}{2} \operatorname{Re}\left\langle q^{*}, Q_{W_{20} \bar{q}}\right\rangle+\frac{1}{2} \operatorname{Re}\left\langle q^{*}, C_{q q \bar{q}}\right\rangle .
\end{aligned}
$$

Since $\alpha_{1}^{\prime}\left(b_{n,+}^{H}\right)>0$, the bifurcation is forward (backward) and the bifurcating periodic solutions on the center manifold are orbitally asymptotically stable (unstable) if $\operatorname{Rec}_{1}\left(b_{n,+}^{H}\right)<0(>0)$.

\section{References}

[1] P. Gray and S. K. Scott, Autocatalytic reactions in the isothermal continuous stirred tank reactor: isolas and other forms of multistability, Chem. Eng. Sci. 38 (1983), 29-43.

[2] P. Gray and S. K. Scott, Autocatalytic reactions in the isothermal, continuous stirred tank reactor: oscillations and instabilities in the system $A+2 B \rightarrow 3 B, B \rightarrow C$, Chem. Eng. Sci. 39 (1984), 1087-1097.

[3] B.Peña and C.Pérez-García, Stability of Turing patterns in the Brusselator model, Phys. Review E, 64(2001)5:056213.

[4] G. Dangelmayr, Degenerate bifurcation near a double eigenvalue in the Brusselator, J. Austral. Math. Soc. Ser. B, 28 (1987) 486-535.

[5] K. J. Brown and F. A. Davidson, Global bifurcation in the Brusselator system, Nonlinear Analysis:TMA, 24(1995)1713-1725.

[6] M. Ashkenazi and H.G.Othmer, Spatial patterns in coupled biochemical oscillators, J. Math. Biology, 5(1978)305-350.

[7] R. Peng and M. Wang, Pattern formation in the Brusselator system, J. Math. Anal. Appl. 309 (2005)151-166.

[8] J. G. Auchmuty, G. Nicolis, Bifurcation analysis of nonlinear reaction-diffusion equations-I. Evolution equations and steady state solutions, Bull. Math. Biol. 37 (1975) 323-365.

[9] R. Peng and M. Wang, On steady-state solutions of the Brusselator-type system, Nonliear Analysis:TMA, 71(2009)1389-1394.

[10] S. L. Hollis, R. H. Martin and M. Pierre, Global existence and boundedness in reactiondiffusion systems, SIAM J. Math. Anal. 18 (3) (1987) 744-761.

[11] T. Kolokolnikov,T. Erneux and J.Wei, Mesa-type patterns in the one-dimensional Brusselator and their stability, Phisical D, 214(2006) 63-77.

[12] H. Kitano, Systems biology: a brief overview, Science, 295 (2002) 1662-1664.

[13] P. Gormley, K. Li, and G. W. Irwin, Modeling molecular interaction pathways using a twostage identification algorithm, Systems and Synthetic Biology, 1 (2007) 145-160. 
[14] I. Schreiber and M. Marek, Strange attractors in coupled reaction-diffusion cells, Physica D, 5 (1982)258-272.

[15] J. J. Tyson, K. Chen and B. Novak, Network dynamics and cell physiology, Nature Reviews: Molecular Cell Biology, 2 (2001) 908-916.

[16] Y. C. You, Global attractor of a coupled two-cell Brusselator model, arXiv:0906.4345v1.

[17] J. Zhou and C. Mu, Pattern formation of a coupled two-cell Brusselator model, J. Math. Anal. Appl. 366 (2010) 679-693.

[18] F. Yi, J. Wei and J. Shi, Bifurcation and spatio-temporal patterns in a diffusive homogeneous predator-prey system, J. Diff. Eqns. 246 (2009) 1944-1977.

[19] F. Yi, J. Liu and J. Wei, Spatiotemporal pattern formation and multiple bifurcations in a diffusive bimolecular model, Nonlinear Analysis: RWA. 11 (2010) 3770-3781.

[20] B. D. Hassard, N. D. Kazarinoff and Y. H. Wan, Theory and applications of Hopf Bifurcation, Cambridge University Press, Cambridge, 1981.

[21] M. G. Crandall and P. H. Rabinowitz, Bifurcation, perturcation of simple eigenvalues and linearized stability, Arch. Ration. Mech. Anal. 52(1973)161-180.

[22] J. Shi and X. Wang, On global bifurcation for quasilinear elliptic systems on bounded domains, J. Diff. Eqns. 246(2009)2788-2812.

Department of Mathematics, Harbin Institute of Technology, Harbin, Heilonguiang, 150001, P.R.China; School of Mathematics and Computational Science, China University of Petroleum (East China), Qingdao, 266555, P. R. China

Department of Mathematics, Harbin Institute of Technology, Harbin, Heilonguang, 150001, P.R.CHINA

E-mail address: weijj@hit.edu.cn 